Vol. 15, $\mathbf{N}^{\circ} 1$ | 1 er semestre 2021 | 2021

Logique algorithmique et reproduction sociétale: les médiations sociales saisies par les algorithmes

\title{
Dix petits hacks Tinder : les algorithmes au service d'une économie spéculative des rencontres amoureuses et sexuelles
}

Ten Little Tinder Hacks: Algorithms in Service of a Speculative Dating and Hookup Economy

Diez pequeños hacks Tinder : los algoritmos al servicio de una economía especulativa de las citas amorosas y sexuales.

David MYLES et Martin BLAIS

\section{(2) OpenEdition}

Journals

Édition électronique

URL : https://journals.openedition.org/ticetsociete/5829

DOI : 10.4000/ticetsociete.5829

Éditeur

Association ARTIC

Édition imprimée

Pagination : 97-125

Référence électronique

David MYLES et Martin BLAIS, «Dix petits hacks Tinder : les algorithmes au service d'une économie spéculative des rencontres amoureuses et sexuelles », tic\&société [En ligne], Vol. 15, N 1 | 1er semestre 2021 | 2021, mis en ligne le 20 mai 2021, consulté le 22 mai 2021. URL : http:// journals.openedition.org/ticetsociete/5829; DOI : https://doi.org/10.4000/ticetsociete.5829 
Dix petits hacks Tinder : les algorithmes au service d'une économie spéculative des rencontres amoureuses et sexuelles

David Myles

myles.david@uqam.ca

Martin Blais

blais.martin@uqam.ca 
Dix petits hacks Tinder : Les algorithmes au service d'une économie spéculative des rencontres amoureuses et sexuelles

\title{
Dix petits hacks Tinder : les algorithmes au service d'une économie spéculative des rencontres amoureuses et sexuelles
}

\begin{abstract}
David Myles détient un doctorat en communication et est professeur associé au département de sexologie de l'Université du Québec à Montréal. Ses travaux portent sur les enjeux socioculturels et politiques engendrés par les médias numériques. II a codirigé l'ouvrage Méthodes de recherche en contexte numérique paru en 2020 et poursuit actuellement un séjour de recherche postdoctorale à l'Institute for Gender, Sexuality, and Feminist Studies de l'Université McGill. myles.david@uqam.ca
\end{abstract}

Martin Blais détient un doctorat en sociologie et est professeur titulaire au département de sexologie de l'Université du Québec à Montréal. Ses travaux portent notamment sur la diversification des biographies sexuelles et relationnelles, ainsi que ses conditions sociales et culturelles d'émergence. II est titulaire de la Chaire de recherche sur la diversité sexuelle et la pluralité des genres et a codirigé l'ouvrage Intimités et sexualités contemporaines paru en 2020. blais.martin@uqam.ca 
David Myles et Martin Blais

\section{Dix petits hacks Tinder : les algorithmes au service d'une économie spéculative des rencontres amoureuses et sexuelles}

Résumé : La médiation des rencontres amoureuses et sexuelles repose sur une longue tradition de services-conseils. Cet article analyse un corpus de guides-conseils développés autour de l'application populaire Tinder. Notre analyse suggère que la pratique de blackboxing algorithmique, soit le fait de caractériser les algorithmes à titre de boîtes noires, est centrale aux guides étudiés. Ces guides misent sur l'opacité présumée des algorithmes de Tinder pour vanter certains produits, conseils ou services visant la facilitation des rencontres. La pratique de blackboxing algorithmique renforce principalement l'injonction de hacker Tinder. Dans ce contexte, la notion de hack renvoie à l'importance pour les usagers de moduler leurs comportements afin de les rendre plus algorithmiquement intelligibles, notamment en fonction de leur genre. Ainsi, nous suggérons que les algorithmes de Tinder participent à reproduire une économie spéculative des rencontres amoureuses et sexuelles.

Mots-clés : algorithmes, applications de rencontres, boîtes noires, économie spéculative, services-conseils.

\section{Ten Little Tinder Hacks: Algorithms in Service of a Speculative Dating and Hookup Economy}

Abstract: The mediation of love and sexuality has often relied on dating advice. This paper analyses a corpus of dating guides concerning the popular app Tinder. Our analysis suggests that the practice of algorithmic blackboxing, that is, characterizing algorithms as black boxes, is central to these guides that rely on the alleged opacity of Tinder's algorithms to praise certain products, advice, or services that aim to facilitate matchmaking. The practice of algorithmic blackboxing primarily encourages users to hack Tinder. In this context, the notion of hack stresses the importance for users to modulate their behaviours in ways that make them more algorithmically recognisable, especially in relation to their gender. 
Dix petits hacks Tinder : Les algorithmes au service d'une économie spéculative des rencontres amoureuses et sexuelles

Thus, we suggest that Tinder's algorithms participate in reproducing a speculative dating economy.

Keywords: algorithms, black box, dating advice, dating apps, speculative economy.

\section{Diez pequeños hacks Tinder : los algoritmos al servicio de una economía especulativa de las citas amorosas y sexuales}

Resumen: La mediación en las citas y encuentros sexuales a través de servicios de consultoría goza de una larga tradición. Este artículo analiza un corpus de guías-consejos desarrollados en el entorno de la popular aplicación Tinder. El análisis sugiere que la práctica del blackboxing algorítmico, es decir, el hecho de caracterizar a los algoritmos como cajas negras, es fundamental en las guías estudiadas. Estas guías utilizan la supuesta opacidad de los algoritmos de Tinder para promocionar ciertos productos, consejos o servicios destinados a facilitar las citas. La práctica del blackboxing algorítmico refuerza la posibilidad de hackear Tinder. En este contexto, la noción de hack hace que los usuarios modulen sus comportamientos, de forma que puedan hacerlos más inteligibles algorítmicamente; y esto de acuerdo con su género. Finalmente, se concluye que los algoritmos de Tinder tienden a reproducir una economía especulativa de las citas amorosas y los encuentros sexuales.

Palabras clave: algoritmos, aplicaciones de citas, caja negra, consultoría de citas, economía especulativa. 
David Myles et Martin Blais

\section{Introduction}

La médiation des rencontres amoureuses et sexuelles repose sur une longue tradition de conseils déployés à travers des maximes populaires ou des manuels de pédagogie amoureuse (Luhmann, 1986), des tribunes radiophoniques ou téléphoniques (Cardon, 2003), ainsi que des courriers du cœur ou des émissions télévisées de confidences (Bänziger et Duttweiler, 2006). Ces conseils répondent aux préoccupations et inquiétudes engendrées par les transformations contemporaines des modalités sociales de rencontre, notamment en regard des meilleures manières de vivre sa vie, des "lois " universelles de l'attraction, des "bonnes" stratégies pour rencontrer l'âme sœur ou des attentes alléguées chez les partenaires potentiels. Autour de ces préoccupations se constitue un marché de services-conseils visant à rétablir des repères normatifs quant aux relations intimes (Béjin et Pollack, 1977; Giddens, 1992; Illouz, 2012). Pour justifier leur bien-fondé, ces services s'appuient entre autres sur des recherches dites scientifiques visant à percer les mystères de l'attraction entre partenaires ou à déterminer les caractéristiques contribuant à faire durer les couples, comme la compatibilité.

Aujourd'hui, l'expertise proclamée de l'intime se traduit autant dans le développement de sites et d'applications mobiles de rencontres que dans le foisonnement de conseillers prétendant détenir des connaissances privilégiées. D'une part, les technologies numériques de rencontres ont professionnalisé les techniques d'appariement, mettant au point des algorithmes prétendant estimer la probabilité d'intérêt et d'attraction mutuels entre partenaires potentiels. D'autre part, invoquant la complexité et l'opacité de ces algorithmes, des experts cherchent à monétiser leurs " connaissances" en livrant leurs conseils aux usagers. On assisterait ainsi à une double rationalisation de la rencontre: l'assignation d'un caractère rationnel et scientifique aux technologies numériques qui introduiraient une logique de la raison dans une sphère généralement associée à la déraison et à la passion; et le souci de rationaliser la recherche de partenaire, de la rendre efficace en promettant une relation épanouissante à 
Dix petits hacks Tinder : Les algorithmes au service d'une économie spéculative des rencontres amoureuses et sexuelles

condition de savoir tirer profit des algorithmes nouvellement mis en place.

Dans cet article, nous analysons les services-conseils développés en périphérie de l'application Tinder pour mieux comprendre la médiation algorithmique de l'amour et de la sexualité (et de leur marchandisation) et leurs implications pour les cultures contemporaines. Spécifiquement, nous mobilisons la notion de blackboxing algorithmique (Bucher, 2018) afin d'examiner le rôle des algorithmes dans la reproduction d'une économie spéculative des rencontres amoureuses et sexuelles commensurables aux logiques capitalistes du marché.

\section{Au-delà de la boîte noire: la pratique du blackboxing algorithmique}

La notion d'algorithme a fait l'objet d'un nombre croissant de définitions durant la dernière décennie et demeure fortement polysémique dans les sciences humaines et sociales (Kitchin, 2017). Si, en informatique, les algorithmes réfèrent à des "procédures encodées servant à transformer des données entrantes en un résultat désiré en se basant sur des calculs spécifiques » (Gillespie, 2014, p. 167), cette définition techniciste est souvent critiquée pour son caractère simpliste qui ne permettrait pas de cerner l'ampleur et la portée des implications culturelles, politiques et économiques des algorithmes pour les sociétés contemporaines. Gillespie (2016, p. 22) propose une définition plus complexe des algorithmes, à titre d'ensembles sociotechniques « contribuant à la production de connaissances et à la prise de décisions [au sein desquels] les gens, représentations et informations sont transformés en données, sont placés dans des relations systématiques/mathématiques et se voient attribuer une valeur basée sur une évaluation calculée ». En somme, l'intérêt des chercheurs en sciences sociales et humaines pour les algorithmes ne se situe pas nécessairement dans leurs opérations computationnelles à proprement parler, mais dans l'imbrication de procédures algorithmiques dans le développement de savoirs et dans l'accomplissement d'expériences sociales. 
Bucher (2018) rappelle l'importance de considérer l'ontologie politique des algorithmes qui repose sur leur caractère performatif, événementiel et itératif. Pour l'auteure, il ne suffirait pas de saisir ce que les algorithmes sont, mais aussi ce qu'ils font dans les contextes sociotechniques où ils sont déployés. L'ontologie politique des algorithmes réfère à leur état perpétuel d'émergence et à la possibilité pour eux d'exercer une agentivité plus ou moins importante selon les contextes, notamment en matière de gouvernementalité (leur rôle dans la modulation des comportements et attitudes des sujets) et d'individuation (leur rôle dans la genèse sociotechnique des sujets même) (Rouvroy et Berns, 2013). Ainsi, dans la lignée des approches foucaldiennes sur le pouvoir (Foucault, 1975), Bucher (2018) soutient que la dimension politique des algorithmes ne serait pas strictement répressive, mais aussi productive. Le mode d'existence des algorithmes reposerait précisément sur leur capacité à exercer une agentivité dans ou sur les cultures contemporaines.

Afin d'examiner le caractère agentif des algorithmes, un nombre croissant d'auteurs s'intéressent aux interactions qu'entretiennent les usagers avec des processus d'automation. Par exemple, en se basant sur une analyse politique étatsunienne, Gillespie (2014) a développé la notion d'intelligibilité algorithmique pour désigner les tactiques développées par les usagers qui adaptent leurs activités en ligne afin de les rendre plus facilement repérables par les moteurs de recherche et de gagner en visibilité. De façon similaire, Velkova et Kaun (2019) ont proposé le concept de résistance algorithmique afin de rendre compte des tactiques de réparation développées par les usagers qui cherchent à contrer et éventuellement compenser les biais reproduits par les processus d'automation opérés par les plateformes numériques.

Si la définition et la portée des algorithmes génèrent d'importants débats intellectuels et politiques, les modalités de leur problématisation - c'est-à-dire la manière dont ils sont construits à titre de problème social (Foucault, 1994) - ne semblent pas susciter autant de contentions. En effet, les algorithmes sont presque unanimement problématisés selon leur propension à opérer sous divers degrés d'opacité et de secret (Burrell, 2016). Ils joueraient un rôle central dans la mise en place de stratégies industrielles 
Dix petits hacks Tinder : Les algorithmes au service d'une économie spéculative des rencontres amoureuses et sexuelles

d'obscurcissement (Ananny et Crawford, 2018; Pasquale, 2015) et leur mode de conception reproduirait de facto une asymétrie de pouvoir entre développeurs et usagers (Bucher, 2018). Les frontières ontologiques des algorithmes seraient difficiles à cerner étant donné leur caractère réticulaire et distribué (Neyland et Möllers, 2017), ce qui participerait également à leur impénétrabilité (Dourish, 2016). Qui plus est, les algorithmes opérés par les médias et technologies numériques contribueraient à la reproduction d'inégalités, notamment en matière de classe sociale (Eubanks, 2018), d'appartenance ethnoculturelle (Noble, 2018) et de genre (Massanari, 2017).

La problématisation des algorithmes semble ainsi reposer sur une série de considérations causales. D'abord, les algorithmes sont des dispositifs complexes qui sont difficiles à saisir. Ensuite, ces dispositifs génèrent d'importants enjeux culturels, politiques et économiques. Enfin, les difficultés liées à l'analyse des algorithmes sont d'autant plus préoccupantes que les enjeux qu'ils suscitent pour les sociétés contemporaines sont de grande ampleur.

Pour Bucher (2018), cette façon de problématiser les algorithmes repose sur l'emploi d'une stratégie discursive qu'elle nomme le blackboxing, à savoir que le problème social que posent les algorithmes est construit en caractérisant ceux-ci comme des boîtes noires, dont l'opacité et l'impénétrabilité rendent difficile tout examen approfondi (pour les chercheurs, les politiciens, les praticiens, les militants, etc.). Pour l'auteure, l'attrait du blackboxing ne se limite pas à statuer si les algorithmes constituent (ou non) des boîtes noires en tant que telles; il permet aussi de comprendre les motivations des acteurs à les problématiser ainsi. Caractériser les algorithmes comme des boîtes noires servirait à orienter les débats politiques vers des enjeux d'imputabilité et de transparence algorithmique et à formuler des revendications (Pasquale, 2015). Par exemple, le blackboxing est fréquemment employé par les militants qui exigent une plus grande imputabilité de la part des géants du Web face aux procédés algorithmiques qu'ils opèrent. Inversement, les propriétaires de plateformes mobilisent la même stratégie pour se dédouaner de leurs responsabilités quant aux inégalités engendrées par les procédés d'automation qu'ils mettent en place. 
En ce sens, la question n'est pas de savoir «si nous pouvons connaître les algorithmes, mais quand son domaine d'intelligibilité est rendu plus ou moins probable [...] c'est-à-dire quand les algorithmes sont cadrés comme des inconnus, pour qui et pour quelle raison » (Bucher, 2018, p. 47). II ne suffirait pas de réifier l'algorithme-comme-boîte-noire à titre de chose, mais de se concentrer sur la pratique de blackboxing « qui consiste à donner l'impression d'une boîte stable en premier lieu » (Bucher, 2018, p. 64). Ce faisant, Bucher (2018, p. 47) affirme que « ce qui doit être examiné de manière critique, alors, n'est pas nécessairement le contenu caché de la boîte, mais les pratiques politiques et sociales mêmes qui aident à maintenir la notion d'algorithmes à titre de boîtes noires ".

L'un des principaux attraits du blackboxing renvoie à sa capacité de construire l'algorithme à titre " d'inconnu stratégique » (McGoey, 2012), c'est-à-dire en tant qu'objet que l'on ne peut entièrement déchiffrer et qui est précisément mobilisé en raison de son caractère soi-disant indéchiffrable. Cette inintelligibilité présumée constituerait l'un des principaux modes heuristiques utilisés pour attribuer un sens aux algorithmes, à savoir qu'il serait très difficile, voire impossible de les saisir dans leur entièreté. Conséquemment, les algorithmes agissent à titre d'alibis épistémiques (McGoey, 2012), dans la mesure où ils permettent de mobiliser stratégiquement l'absence présumée de connaissances sur un sujet donné (ici, les algorithmes et les effets qu'ils entraînent) afin d'acquérir la légitimité nécessaire pour se positionner autoritairement face au sujet en apparence inintelligible (ou pour s'en dissocier).

Pour la présente étude, nous examinons par quels discours certains acteurs caractérisent les algorithmes de Tinder en tant que boîtes noires et relevons les implications que ces pratiques discursives génèrent pour l'industrie des rencontres. Pour ce faire, nous mobilisons des exemples tirés de guides-conseils sur Tinder publiés en ligne sous forme d'articles par des experts autoproclamés et d'autres commentateurs. Soixante articles (traduits de l'anglais au français) ont été initialement sélectionnés via une recherche par mots-clés sur Google et 48 ont fait l'objet d'une analyse du discours d'inspiration poststructuraliste menée à partir du logiciel QDA Miner. Ce type d'analyse discursive se 
Dix petits hacks Tinder : Les algorithmes au service d'une économie spéculative des rencontres amoureuses et sexuelles

concentre sur les cadres régulateurs qui gouvernent les discours, ceux qui font des discours des énoncés standardisés et autoritaires participant à produire des sujets ou des problèmes sociaux et à en tirer du sens (Graham, 2011). Les guides sélectionnés ont été publiés de 2015 à 2019, principalement sur des blogues et sites Web divers (35), des magazines en ligne (7), des forums (4) et des journaux en ligne (2). Ils ont été colligés en septembre 2019 selon deux critères : ils devaient offrir aux lecteurs des conseils quant aux meilleurs usages de Tinder et aborder les algorithmes opérés par l'application de rencontres. Les prochaines sections présentent les résultats de notre analyse.

\section{L'injonction de hacker Tinder}

Lancé en 2012, Tinder se targue d'être l'application de rencontres la plus populaire au monde en revendiquant plus de 50 millions d'utilisateurs. Sur Tinder, chaque usager est invité à remplir un profil composé d'une brève biographie et d'un album photo, ainsi que des contenus issus d'autres plateformes numériques, comme Facebook, Instagram ou Spotify. L'application met peu l'accent sur les caractéristiques sociodémographiques de ses usagers ou sur leurs préférences, outre le genre, l'âge, l'orientation sexuelle et la distance géographique. Chaque usager est plutôt invité à passer à travers une liste prédéterminée de profils. Pour sélectionner un profil, on le fait glisser avec le doigt vers la droite; pour le rejeter, on le fait glisser vers la gauche. La sélection des profils suggérés à chaque usager est donc prise en charge par des algorithmes de classement et de recommandation réputés comme étant sophistiqués. Lorsque deux usagers sélectionnent leur profil respectif, un canal de messagerie privée s'ouvre et les invite à interagir.

L'opacité présumée des algorithmes mis en place par Tinder donne lieu à une importante économie spéculative des rencontres amoureuses et sexuelles. Avec ses millions d'usagers, comment l'application sélectionne-t-elle les profils qu'elle suggère ou dissimule à autrui? Dans les guides étudiés, il est généralement présumé que Tinder fonctionne (ou fonctionnait) selon la méthode d'évaluation Elo, développée à l'origine pour classer les joueurs de tournois d'échecs (Krüger et Spilde, 2019). L'application accorderait 
un score de désirabilité à chaque usager en calculant l'engouement qu'il suscite chez les autres. Ainsi, l'application recommanderait aux usagers les profils jugés symétriquement populaires. Les profils en forte demande seraient proposés aux usagers qui sont aussi en forte demande, tandis que les usagers atterrissant au bas de cette stratification se matcheraient entre eux. S'il est difficile de confirmer la véracité de ces informations, ce sont les tentatives d'explicitation des algorithmes de Tinder qui constituent notre matériau d'analyse, et non les algorithmes mêmes.

Hautement spéculatifs, les guides-conseils étudiés opèrent sous la logique générale voulant que, si les usagers connaissaient mieux les procédés algorithmiques de l'application, ils pourraient obtenir plus de matchs. Ainsi, ces guides présentent Tinder comme une boîte noire dont il faut percer les secrets pour en maximiser l'usage. Cette stratégie discursive sert d'abord à attirer le lecteur en réifiant les procédés algorithmiques de Tinder en un algorithme unique au caractère "magique ", " mystérieux " et " difficile à expliquer ». Comme on l'indique dans l'un des guides étudiés : "Les utilisateurs ne savent pas comment ce monstre fonctionne dans le détail ». Le fait d'invoquer l'inintelligibilité présumée de Tinder permet ensuite de positionner l'auteur du guide comme un expert ou un conseiller prêt à offrir des stratégies inédites au lectorat. Dans les guides, il est fréquent de lire des titres comme "Les algorithmes révélés! » ou "Conseils d'un mec qui a déchiffré l'algorithme de Tinder! ». Le blackboxing algorithmique opère en misant sur l'importance pour les usagers d'acquérir "une connaissance plus approfondie de l'industrie des applications de rencontres et de ses coulisses » et d'accéder aux « règles secrètes de Tinder ».

L'équipe Tinder participe également à entretenir un certain mysticisme autour des procédés algorithmiques qu'elle met en place. La compagnie a notamment publié un billet de blogue en 2019 intitulé "Powering Tinder - The Method Behind Our Matching " qui évoque la méthode utilisée afin de jumeler les usagers, sans toutefois livrer tous les détails techniques. Ce type d'annonce n'est pas seulement informatif. En offrant un morceau du casse-tête aux usagers, Tinder participe à soutenir la spéculation dans une industrie secondaire qui se construit autour de l'application (et qui inclut entre autres les guides-conseils que nous avons 
Dix petits hacks Tinder : Les algorithmes au service d'une économie spéculative des rencontres amoureuses et sexuelles

analysés). À partir de ces indices, les conseillers spéculent sur les stratégies à mettre en place pour tirer avantage de l'application. Prenons ces deux extraits d'un guide où l'auteur décrit Tinder :

\footnotetext{
Ces hacks Tinder peuvent aider les losers à obtenir des matchs

À première vue, Tinder semble être un site de rencontres assez simple. Ceci est vrai seulement de l'extérieur, car à l'intérieur de l'application existe un algorithme plutôt sophistiqué. Cet algorithme est responsable des matchs entre usagers et vous classifie comme étant désirables ou indésirables. Mais comme tout code informatique, il peut être hacké et exploité. Après avoir compris comment il fonctionne, vous pourrez l'utiliser à votre avantage. [...]

Ce n'est pas tout le monde qui trouve facile de naviguer dans le monde de Tinder. II s'agit d'une application trompeusement complexe, et il existe une manière très spécifique par laquelle l'algorithme de l'application vous classe et vous matche. Pour certains, le fait de ne recevoir aucun match peut générer du désespoir et vous rendre encore plus désespéré que vous ne l'étiez avant d'avoir utilisé Tinder. Mais il existe plusieurs façons d'utiliser cet algorithme pour hacker l'application, pour augmenter votre score et garantir plusieurs matchs et même des rencarts. Certains de ces hacks sont basés sur de la logique pure. D'autres considèrent la manière dont l'application fonctionne sur un niveau très technique. Tous ces hacks sont faciles à comprendre et amélioreront votre acuité Tinder plus que jamais auparavant!
}

Figure 1. Les algorithmes de Tinder comme boîtes noires

Après avoir qualifié l'algorithme Tinder de boîte noire, les auteurs utilisent souvent la métaphore du hack ou son équivalent afin d'orienter les lecteurs vers des stratégies pour faire face aux problèmes qu'ils ont eux-mêmes construits discursivement. On parle dans ce cas-ci de l'importance de "truquer le système », de " manipuler l'algorithme " ou d'utiliser des " codes de triche ». En ce sens, les hacks Tinder sont présentés comme " une manière facile de maximiser vos capacités de matching » ou encore de faire en sorte que « les algorithmes travaillent pour vous".

Dans les cultures populaires, la notion de hack est habituellement utilisée pour désigner une "prouesse informatique " qui est déployée dans une visée subversive de détournement technologique, notamment à des fins politiques, financières, frauduleuses ou humoristiques (Bardini et Proulx, 2002). Dans les cultures numériques émergentes, la notion de hack est parfois associée à l'économie du clickbait qui mise sur la diffusion de vidéos ou articles sensationnalistes servant à attirer les clics d'internautes et dont une itération très populaire repose sur l'élaboration de life hacks pour faciliter la vie quotidienne. Dans les guides analysés, le 
hack Tinder est présenté comme une sorte de stratégie de déboîtement algorithmique visant à maîtriser les algorithmes ou les fonctionnalités de l'application.

Les hacks proposés touchent principalement trois dimensions des procédés algorithmiques prétendument mis en place par Tinder: le score de désirabilité, la fréquence des activités sur l'application et les préférences personnelles. Ces hacks puisent largement dans les indices fournis par l'équipe Tinder, mais aussi dans des expériences et anecdotes personnelles. En découle une série de conseils basés sur des propositions et hypothèses plus ou moins fondées. Pour augmenter son score de désirabilité, il suffirait de changer ses photos et sa biographie dans le but de les rendre plus attrayantes (nous reviendrons sur ce point plus bas). Pour jouir d'une plus grande désirabilité, il suffirait d'utiliser l'application quotidiennement tout en faisant preuve de discernement dans la sélection des profils d'autrui (il ne faudrait surtout pas accepter tous les profils suggérés, mais il serait primordial de répondre à chacun de nos matchs!). L'application déploierait également des procédés d'apprentissage computationnel qui proposeraient des profils selon nos habitudes ou préférences personnelles de consommation - tirées des profils Facebook, Instagram ou Spotify associés au compte Tinder - ou selon notre historique de sélection de profils (il faudrait donc demeurer cohérent face aux profils que nous sélectionnons!).

En ce sens, la notion de hack Tinder ne fait pas référence à la possibilité de contourner ou de subvertir les algorithmes de l'application. Au contraire, les guides affirment généralement qu'il serait crucial pour les usagers de moduler leurs propres pratiques et comportements afin de les rendre plus algorithmiquement intelligibles, pour reprendre l'expression de Gillespie (2016). Cette modulation comportementale est identifiée comme étant la meilleure façon pour les usagers de maximiser le potentiel de Tinder et d'éviter les conséquences associées à tout manquement. Comme l'indique un auteur : "Tinder vous accorde des points pour votre bonne conduite et les confisque si vous vous conduisez mal ». Un autre soutient que « si vous glissez trop à droite, l'algorithme de Tinder va se retourner contre vous ". De façon similaire, ce guide propose des mises en garde à ses lecteurs : 
Dix petits hacks Tinder : Les algorithmes au service d'une économie spéculative des rencontres amoureuses et sexuelles

\section{9 hacks Tinder que vous devriez définitivement essayer}

Tinder utilise un algorithme qui décide qui verra votre profil et quels profils vous seront proposés. Cela s'effectue en vous assignant un score Elo [...]. Ceci signifie que Tinder a créé un ensemble de règles à travers lesquelles votre attractivité est évaluée, ce qui ne réfère pas seulement à votre apparence. II existe des règles comportementales. Et si vous ne les suivez pas? Vous risquez de diminuer votre score et, en conséquence, votre visibilité, ce qui veut dire que vous aurez moins de matchs. Si vous faites une erreur banale ou si vous brisez une règle majeure, vous pourriez même être secrètement banni. Votre profil sera caché sans notification. Vous serez quand même en mesure de voir les profils, mais personne ne pourra vous voir, vous laissant sans match.

Figure 2. L'injonction de hacker Tinder

Les guides Tinder analysés reflètent l'existence autant qu'ils participent à la constitution d'un dispositif algorithmique servant à discipliner les usagers (Foucault, 1975). Ce dispositif disciplinaire repose sur une logique à la fois verticale (l'équipe Tinder met en place des fonctionnalités et procédés d'automation visant la modulation de comportements) et latérale (les algorithmes sont nourris par les activités des usagers-mêmes qui sont appelés à se surveiller entre eux en punissant les comportements d'autrui jugés déviants). Conséquemment, l'injonction de hacker Tinder se résume à bien comprendre les impératifs de l'application pour mieux s'y plier. Dans ce cas, il devient difficile de discerner si les hacks proposés font en sorte que les algorithmes travaillent pour les usagers ou s'ils incitent plutôt les usagers à modifier leurs comportements pour faciliter la tâche des algorithmes.

Le caractère disciplinaire des algorithmes est apparu particulièrement évident dans les conseils concernant le choix des photos. Selon les guides étudiés, les photos de profil semblent jouer un rôle central dans la présentation de soi et l'expression identitaire sur l'application: "Tinder est d'abord une expérience visuelle ». À ce titre, l'ensemble des conseils formulés présente un certain paradoxe. D'un côté, ils rappellent presque unanimement l'importance de faire preuve d'authenticité dans les photos et dans les interactions avec autrui. II importerait d'être "honnête ", " naturel », " sincère » et " décontracté », ainsi que de fournir des informations « fidèles à la réalité ». De l'autre côté, cette authenticité est calculée ou performée afin de se mettre en valeur, ce qui 
David Myles et Martin Blais

représente pour Tinder un problème potentiel de surenchère généralisée (Duguay, 2017).

Au sujet de la performance calculée de l'authenticité, un auteur explique : "II s'agit de montrer aux gens à quoi s'attendre avec vous. II faut prendre un risque et vraiment montrer aux autres la meilleure version de ce qu'ils vont recevoir lorsqu'ils vous rencontreront ». Ces hacks visuels sur Tinder se répercutent aussi à l'extérieur de l'application. Afin de montrer le meilleur de soi-même sans avoir l'air malhonnête, certains guides suggèrent simplement d'intégrer les habitudes jugées désirables au quotidien. Vantant les mérites d'exposer des photos où l'on se montre physiquement actif, un conseiller invite à « revoir son profil et sa bio pour faire des petits ajustements [...], mais assurez-vous d'intégrer aussi ces ajustements dans votre vie ». Ainsi, on propose comme hack de changer ses propres habitudes quotidiennes pour que son profil soit jugé plus attirant et puisse tirer profit des algorithmes de recommandation, tout en restant fidèle à la réalité. Ce faisant, ces guides incitent à uniformiser les pratiques sur Tinder en insistant sur le caractère disciplinaire de l'application qui pénaliserait les usagers qui ne s'y conforment pas.

\section{La reproduction d'une économie spéculative des rencontres amoureuses et sexuelles}

Les guides que nous avons analysés sont généralement développés pour proposer des produits et services associés à la recherche de partenaires. Certains de ces services sont intégrés à Tinder. Des guides vantent l'utilité des fonctionnalités payantes permettant de contourner les limites imposées par les algorithmes. Par exemple, la fonctionnalité Passport permet de dépasser les limites géographiques imposées aux usagers, alors que Rewind dévoile les profils rejetés antérieurement. Ces conseils agissent comme des infopublicités qui présentent les différents produits Tinder et illustrent leur potentiel pour le lecteur. Certains auteurs mentionnent par ailleurs qu'ils auraient pu toucher une commission pour la rédaction de leur article, évoquant une possible transaction financière avec la compagnie.

De nombreux autres services périphériques sont aussi proposés au lectorat. Par exemple, plusieurs guides conseillent de «sous- 
Dix petits hacks Tinder : Les algorithmes au service d'une économie spéculative des rencontres amoureuses et sexuelles

traiter la sélection de photos " à des sites Web et applications tierces, comme Photofeeler et Askem, qui permettent aux usagers de sonder une foule d'internautes pour évaluer la qualité de leurs photos avant qu'ils ne les utilisent sur Tinder. Ces services cherchent ainsi à reproduire les procédés algorithmiques de stratification de l'application en utilisant une démarche de crowdsourcing. Certains guides conseillent aux usagers de retoucher leurs photos avec l'application payante Facetune pour augmenter leurs matchs. D'autres proposent de faire appel à des services de vérification d'antécédents pour s'assurer de la véracité des informations fournies par leurs partenaires potentiels, ou de requérir à des services de surveillance comme XNSPY pour vérifier si son partenaire actuel utilise Tinder à des fins d'adultère.

En jouant sur le domaine d'intelligibilité des algorithmes, la pratique de blackboxing permet aux experts autoproclamés de se construire une expertise et de revendiquer une autorité autour de la recherche de partenaires. Comme l'indique un auteur : "Bien sûr, le meilleur de tous les hacks demeure de sous-traiter Tinder à des experts en dating. Plus besoin de perdre des heures sur votre téléphone, de sélectionner des profils sans cesse. Laissez-nous nous en occuper - vous devrez simplement vous présenter au rencart ». Ailleurs, un guide vante l'importance des services proposés en misant sur les appréhensions du lectorat : « C'est peutêtre votre profil, mais cela ne signifie pas pour autant que vous êtes la meilleure personne pour le créer ». De manière semblable, un auteur affirme qu' "[il] existe un moyen d'éviter tous ces trucs déprimants, tout ce temps et tous ces efforts! Laissez les experts Tinder de chez $[X]$ tout faire pour vous $"$.

Les guides constituent donc une opportunité d'offrir des services périphériques aux usagers Tinder afin d'augmenter leur capacité à établir des matchs. Ces services peuvent prendre la forme de séminaires ou de séances individuelles avec des coachs en face à face ou en visioconférence, ainsi que des manuels et vidéos payants. "Améliorer votre vie amoureuse avec des conseils pratiques livrés directement dans votre boîte de courriels! ", propose un conseiller. D'autres liens mènent quant à eux vers des groupes sur Facebook et Reddit afin de révéler les secrets de Tinder et de ses algorithmes. Si ces services ne sont pas de facto payants, 
ils s'inscrivent dans une logique de marketing numérique où les consommateurs potentiels sont identifiés à l'aide de cookies et traqués a posteriori sur différentes plateformes. Ces stratégies se sont avérées particulièrement évidentes lorsque les publicités d'agences de rencontres et autres gurus ont été recommandées à l'un des deux auteurs de cet article sur Facebook et YouTube quelques jours après le début de la collecte de données, comme en témoignent les captures d'écran présentées dans la figure 3 .
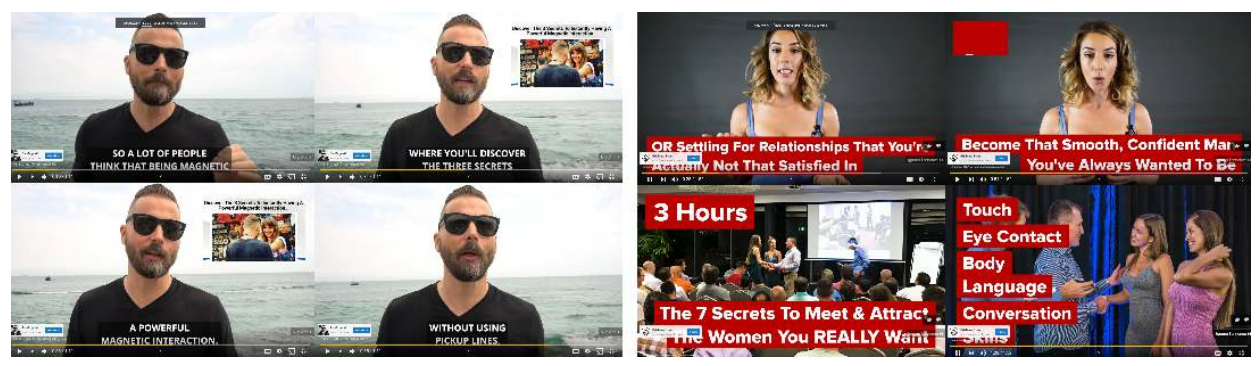

Figure 3. L'économie des rencontres et la surveillance numérique

Certes, l'industrie des rencontres n'est pas récente. Le couplage amoureux ou sexuel a longtemps été médié par des agents humains, médiatiques et technologiques (Bergström, 2019). Néanmoins, l'appification des rencontres génère des enjeux socioculturels et économiques spécifiques, notamment à travers la mise en données des activités d'usagers qui reconfigure les modèles d'affaires et les modes de gouvernance de l'industrie des rencontres (Myles, 2020; Duguay et al., 2017). En ce sens, si l'équipe Tinder cadre ses activités comme appartenant d'abord au domaine des rencontres, l'application est également un outil de collecte de données au service d'une industrie. Ceci est représentatif des mutations contemporaines de l'industrie des rencontres où les dating apps se comportent de plus en plus comme des data apps (Albury et al., 2017).

La mise en données des activités d'usagers se reflète dans le vernaculaire pseudoscientifique employé par les experts-conseils. L'invocation de données et de statistiques semble jouer un rôle 
Dix petits hacks Tinder : Les algorithmes au service d'une économie spéculative des rencontres amoureuses et sexuelles

central dans la construction de leur expertise et la revendication de leur autorité. Une scientifisation des discours s'observe notamment lorsque les auteurs de guides justifient leurs propos en citant des chercheurs se spécialisant dans les logiques de match sur Tinder, travaillant parfois pour la compagnie. "Pour préserver votre santé d'esprit et vos standards ", affirme un guide, "l'experte des rencontres Tinder Darcy Sterling, $\mathrm{PhD}$, vous offre des conseils pour optimiser votre profil Tinder ». S'en suit une liste de statistiques. Comme $72 \%$ des usagers Tinder portent des couleurs neutres, pourquoi ne pas arborer une couleur éclatante sur votre photo principale? On y apprend également que les photos frontales auraient $20 \%$ plus de chance d'être sélectionnées, alors que la sélection d'une photo avec sourire augmenterait les chances de $14 \%$. "On veut savoir que vous êtes prêts à être vulnérables et à montrer votre visage ", clame une experte citée.

Ces extraits illustrent comment la scientifisation des discours sur les rencontres repose largement sur la mise en données des activités d'usagers. Sans la production de ces données, ce type de discours serait difficile à développer. La donnée y devient donc un argument central. Les procédés opérés par les algorithmes de Tinder ne permettent pas seulement la monétisation des activités de leurs membres; ils donnent lieu à un nouveau régime de vérité, c'est-à-dire qu'ils s'inscrivent dans un ensemble d'institutions et de discours contribuant à établir ce qui est considéré comme "vrai » ou « véritable » (Foucault, 2012). Les algorithmes ont déjà été identifiés comme étant les producteurs de nouveaux régimes de vérité reposant sur un mode de gouvernementalité prédictif principalement axé sur la production de big data (Rouvroy, 2014; Ménard et al., 2016). Ceci s'étend à l'industrie des rencontres, où la production d'un grand corpus de données comportementales sert à identifier les "vérités" sur lesquelles les usagers devraient se baser pour moduler leurs activités. Ces "vérités " sont algorithmiquement produites et entrent en compétition (ou s'arriment) avec d'autres régimes de vérité sur l'amour et la sexualité, comme la sagesse populaire, la religion et la science (psychologie clinique, sexologie, biologie évolutive, etc.).

Ces procédés opèrent l'idéologie d'un dataisme qui vante les mérites de la donnée probante à titre de nouvelle heuristique pour 
comprendre et construire les rencontres amoureuses et sexuelles. Comme l'indique van Dijck (2014, p. 198) : « l'idéologie du dataisme témoigne d'une croyance répandue concernant le caractère objectif de la quantification et du monitorage potentiel de toutes sortes de comportements humains et sociaux à travers les technologies numériques ». Les données amassées sont présentées comme étant brutes et objectives, et leur traitement algorithmique est accepté comme étant apte à donner accès à une réalité soi-disant objective (Boyd et Crawford, 2012), négligeant ainsi une panoplie de travaux issus notamment du champ des sciences et technologies qui ont démontré le caractère fabriqué des données (Bowker, 2005).

Malgré les limites et biais inhérents à leur production, les statistiques diffusées par Tinder participent à réimaginer les pratiques idéelles en matière de rencontres amoureuses et sexuelles. À ce titre, les guides étudiés constituent un objet d'analyse particulièrement riche pour saisir ces mutations sur le plan discursif. Notre analyse illustre la reproduction d'une rhétorique circulaire axée sur la donnée. D'abord, Tinder met en place des procédés algorithmiques non neutres qui encouragent les comportements d'usagers jugés appropriés et discriminent ceux jugés déviants. L'application produit et diffuse ensuite des statistiques pour démontrer le succès des membres qui respectent ces règles comportementales. Puis, ces données sont exploitées par toute une industrie périphérique des rencontres (dont les guides que nous avons analysés font partie) qui produit des discours servant à orienter les comportements vers des normes idéelles d'usage, élaborant notamment ces discours sous forme de hacks. Les usagers qui emploient ces hacks sont éventuellement récompensés par des algorithmes qui reconnaissent ces activités comme étant plus compatibles avec les valeurs qu'ils promeuvent. Ce faisant, cette réorientation renforce les statistiques de Tinder voulant que l'obéissance aux impératifs algorithmiques représente la meilleure option pour les usagers esseulés. En somme, cette boucle opère une sorte de prophétie autoréalisatrice misant sur la récursivité des algorithmes. Tinder participe à la fois à construire le problème des usagers et à leur offrir des solutions en mobilisant des arguments tautologiques qui opèrent une idéologie dataiste. 
Dix petits hacks Tinder : Les algorithmes au service d'une économie spéculative des rencontres amoureuses et sexuelles

\section{Les impératifs algorithmiques de l'amour, du genre et de la sexualité}

Le régime de vérité algorithmique instauré par Tinder (entre autres technologies numériques) s'inscrit dans la (re)production de conceptions concernant l'amour, le genre et la sexualité qui sont commensurables avec des logiques capitalistes de marché. À ce titre, la rationalisation des rencontres est rarement cadrée comme étant positive. Certains guides mobilisent les frustrations ressenties face aux applications de rencontres et aux hacks qu'elles requerraient. "Ces trucs font chier, mais ils fonctionnent ", affirme un auteur. Idem pour les algorithmes: les insatisfactions qu'ils génèrent sont mises en opposition à une vision romantique des rencontres. Par exemple, un conseiller affirme : "Le dating : c'est un bordel. Entre les algorithmes, les scores Elo, les filtres de photos, les attentes ruinées, les photos de surf en Thaïlande, les fraudeurs, les plans cul et les rencontres de courte durée, il existerait peut-être quelque chose qui ressemble à l'amour ». Similairement, un auteur interroge ses lecteurs:

\section{5 façons par lesquelles l'algorithme Tinder peut travailler pour vous \\ Vous vous souvenez d'un temps où les médias sociaux étaient simples et n'utilisaient pas d'équations mathématiques ou informatiques? Vous vous souvenez d'un temps où les rencarts étaient simples? D'accord, les rencontres ont toujours été un peu déroutantes, mais le mot 'algorithmes' ne faisait que rarement, voire jamais partie du lexique du monde du dating.}

Figure 4. Les algorithmes et les doléances qu'ils suscitent

Ces extraits illustrent comment certains guides misent sur la croyance voulant que la recherche de l'amour et de la sexualité ait été beaucoup plus simple avant l'introduction des procédés algorithmiques qui, de toute évidence, ne simplifieraient pas les rencontres autant qu'elles les compliqueraient (d'où l'attrait des hacks proposés). II s'agit là d'un autre exemple de blackboxing algorithmique. Pourtant, si les algorithmes constituent des dispositifs relativement récents dans l'industrie des rencontres, la pratique du blackboxing n'est pas nouvelle. En effet, les rencontres amoureuses ont souvent été discursivement construites comme un mystère (Haag, 1992; Luhmann, 1986), une boîte noire dont il faut 
percer les secrets (Cresswell, 2003; Fry, 2015). De la même manière, de nombreux écrits sur les rencontres hétérosexuelles naturalisent l'idée que les hommes et les femmes sont diamétralement opposés (Crawford, 2004), utilisant une forme de blackboxing qui justifie la multiplication de produits dérivés promettant aux hommes et aux femmes une meilleure compréhension mutuelle et un plus grand succès relationnel. L'ouvrage à succès Les hommes viennent de Mars, les femmes viennent de Vénus (Gray, 1992) en est un exemple patent.

De manière générale, les guides étudiés ont tendance à cadrer les rencontres amoureuses et sexuelles sous l'angle de la compétition, du jeu ou du marketing, et à formuler leurs propos en puisant dans des croyances plus ou moins fondées émanant d'une pseudobiologie évolutive. Comme l'indique un conseiller : " Hack Tinder numéro 1 : traitez votre bio comme une publicité ». II suffirait de calquer des stratégies marketing pour trouver un partenaire: « il faut se vendre soi-même " en utilisant un " argumentaire de vente sous pression ». Concernant le choix des photos de profil, on apprend qu'il « existe quelque chose de primal qui fait en sorte que les femmes se pâment devant les hommes qui pratiquent des sports. [...] Les sports avec contact ont tendance à attirer les femmes davantage que les sports sans contact». Ailleurs, on s'exclame: "Messieurs, soyez à l'affût de votre compétition, vos ennemis ». "Bonne chasse! », peut-on lire dans un autre guide. Ou encore : assurez-vous de bien maîtriser "les règles du jeu». L'objectif de ce jeu, de cette compétition, se résumerait à augmenter son score de désirabilité pour générer plus de matchs et, surtout, plus de matchs " de bonne qualité ". "Les femmes sont de plus en plus sélectives sur Tinder » soutient un conseiller. II reviendrait donc " aux mâles de capter l'attention de la femelle » et de "prendre l'initiative ». 
Dix petits hacks Tinder : Les algorithmes au service d'une économie spéculative des rencontres amoureuses et sexuelles

Ces discours reproduisent largement des normes cishétéronormatives régissant la performance du genre et de la sexualité. La forte majorité des lecteurs désignés y sont masculins ${ }^{1}$. Les hommes sont généralement identifiés comme les sujets à changer, ceux qui pourraient bénéficier d'une plus grande " littératie en matière de rencontres ". II appartiendrait aux hommes de prendre soin de leur apparence physique, d'aller régulièrement au gym, de porter la chemise et de maintenir une barbe soignée. Plus important encore, on rappelle constamment aux hommes l'importance de ne pas avoir l'air "louche ». "Les mecs, ne foirez pas tout! », implore un conseiller. Cet accent placé sur l'amélioration de soi s'inscrit pleinement dans les discours caractérisant l'économie des rencontres comme étant intrinsèquement compétitive. "Ce n'est pas une surprise », affirme un auteur, « il existe un nombre disproportionné de mecs sur Tinder, ce qui rend encore plus difficile pour les mâles de notre espèce d'obtenir un match sur l'application ». De manière semblable, on peut lire dans un guide :

Palmarès: Les 11 meilleurs hacks Tinder de tous les temps [Édition 2019!]

Comme les hommes sont trois fois plus nombreux que les femmes sur cette application, les femmes attirantes peuvent se permettre d'être sélectives. Donc, si vous voulez maximiser votre usage de Tinder, vous devez leur montrer que vous êtes mieux que la compétition qui inonde leur boîte de messages.

Figure 5. Tinder, un jeu compétitif

Quant aux femmes, celles-ci figurent parmi les objets difficiles à décoder. À ce titre, un conseiller indique : «Les femmes sont des créatures mystérieusement belles, ne vous avisez pas de les analyser ». De manière générale, les femmes occupent un rôle plus passif dans l'économie des rencontres, où elles sont appelées à

\footnotetext{
${ }^{1}$ Les guides ont été sélectionnés via Google. Pour nous assurer que le moteur de recherche ne discriminait pas nos résultats en fonction de notre genre, nous avons demandé à une collègue de simuler nos recherches en utilisant les mêmes mots-clés. L'ensemble des guides recueillis par la chercheure étaient également rédigés pour un lectorat principalement masculin.
} 
réagir aux avances des hommes. Elles y sont aussi décrites comme des victimes potentielles (de harcèlement, de violence, d'ennui, etc.). Conséquemment, celles-ci devraient être charmées, mais aussi ménagées par leurs prétendants :

10 meilleurs hacks Tinder de 2019 (10x plus de matchs)

Les mecs, prêtez attention. Nous sommes au $21 \mathrm{e}$ siècle et le dating a changé drastiquement. Planifier un rencart avec une fille sur une application exige deux choses : que ce soit public et que ce soit rapide. De nos jours, les filles ont peur pour leur sécurité et leur santé d'esprit lorsqu'elles acceptent le rencart d'un mec sur une application. Elle ne veut pas être kidnappée ni mourir d'ennui dans un rencart interminable.

Figure 6. Tinder et le ménagement des femmes

Les femmes y sont aussi décrites comme des manipulatrices ou des exploiteuses potentielles. Par exemple, on les accuse d'utiliser Tinder pour augmenter leur nombre d'abonnés Instagram. On les dépeint également comme ayant tendance à planifier des rancards dans le but de profiter du portefeuille de leurs prétendants. À l'inverse, les femmes peuvent être perçues comme des ressources lors de l'élaboration du profil. En effet, quoi de mieux qu'une femme pour aider à identifier ce que les femmes recherchent sur l'application? "Les hommes croient peut-être savoir ce que les femmes veulent", avance un des auteurs, "mais sont souvent dans le tort. Ce que tu penses être une photo sexy ou cool peut dans les faits susciter des rires chez une majorité de femmes ". Ainsi, les guides reproduisent la croyance répandue voulant que les femmes représentent des boîtes noires que les usagers masculins doivent hacker pour en révéler les mystères.

En somme, les femmes occupent une place centrale dans les guides étudiés en raison de la valeur alléguée que Tinder leur accorderait. Comme l'affirme un auteur de notre corpus: "Les usagers qui ont le plus de valeur pour Tinder sont les femmes (désirables) qui sont satisfaites et actives sur l'application. Comment Tinder maintient-il les femmes désirables satisfaites? En leur montrant des hommes désirables». Ceci expliquerait l'importance pour les hommes de moduler leurs comportements afin d'améliorer leur score de désirabilité et d'avoir accès à des « filles de bonne qualité ». Tinder en vient donc à transformer la définition 
Dix petits hacks Tinder : Les algorithmes au service d'une économie spéculative des rencontres amoureuses et sexuelles

de ce qui est « désirable » à travers la médiation algorithmique qu'il impose à ses usagers. Comme le suggère un conseiller: "être désirable ne veut pas nécessairement dire être attrayant " sur Tinder. La notion de désirabilité fait référence à la capacité des membres à tirer profit des normes comportementales promues par l'application (rédiger une biographie adéquate, choisir les meilleures photos, indiquer les bons intérêts personnels, faire preuve de discernement lors de la sélection de profils, etc.). II s'agirait d'une désirabilité calculée qui participe à la rationalisation du genre et des rencontres en répondant à des impératifs algorithmiques tant techniques que socioculturels.

\section{Conclusion}

Cet article a proposé une analyse discursive d'un corpus de guides Tinder pour comprendre le rôle des algorithmes dans la reproduction d'une économie spéculative des rencontres amoureuses et sexuelles. D'emblée, il importe de nuancer les résultats de notre étude qui se limite à l'analyse de discours publics, sans toutefois se pencher empiriquement sur les pratiques d'usagers. Si les guides étudiés présentent une série d'injonctions pour hacker Tinder, cela ne signifie pas pour autant que ces injonctions s'incarnent de facto dans les pratiques effectives de chaque usager. De futures recherches pourraient s'intéresser aux stratégies de résistance développées par les usagers face à l'intensification de la médiation algorithmique des rencontres amoureuses et sexuelles. Étant donné le caractère disciplinaire des algorithmes, toute forme de résistance développée à l'intérieur de ces dispositifs serait-elle vouée à l'échec? Le cas échéant, la nonutilisation de ces dispositifs resterait-elle la forme de résistance la plus prometteuse? II apparaît ainsi crucial de s'attarder aux formes d'agentivité que peuvent exercer les acteurs face à ces nouveaux développements sociotechniques.

Notre étude souligne également l'importance d'examiner comment les procédés algorithmiques et d'apprentissage computationnel sont invoqués par divers acteurs cherchant à déployer de nouveaux régimes de vérité, et ce, dans la lignée des travaux portant sur la gouvernance statistique. En ce sens, quels rôles jouent les algorithmes (et les données qu'ils produisent, 
classent, invoquent, obscurcissent, etc.) dans l'élaboration de discours se voulant crédibles et autoritaires? Comment ces discours participent-ils à soutenir des logiques capitalistes de marché? Et comment ces discours pourraient-ils éventuellement être cooptés pour subvertir ces logiques?

Enfin, si les guides Tinder étudiés reproduisent stratégiquement des conceptions largement rationalisées de l'amour, du genre et de la sexualité, ils expriment également des frustrations face à la mise en place d'un système d'évaluation stratifiée de la désirabilité par l'application de rencontres. Soit, la rationalisation des relations amoureuses et sexuelles n'est pas nouvelle (Béjin et Pollack, 1977) et ne se limite pas aux mutations algorithmiques récemment opérées par l'industrie des rencontres. Néanmoins, ces mutations peuvent contribuer à reproduire des conceptions fallacieuses et nocives des relations intimes axées sur la hiérarchisation et la (dé)valorisation systématique de certaines identités genrées ou sexuées. II importe donc de porter un regard critique sur les médiations algorithmiques des rencontres, à l'intersection des études sur la communication, le genre et la sexualité, pour cerner les incidences matérielles et symboliques qu'elles génèrent pour les cultures contemporaines.

\section{Références}

Albury, K., Burgess, J., Light, B., Race, K., et Wilken, R. (2017). Data Cultures of Mobile Dating and Hook-Up Apps: Emerging Issues for Critical Social Science Research. Big Data et Society, 4(2), 1-11. doi: 10.1177/2053951717720950

Ananny, M., et Crawford, K. (2018). Seeing Without Knowing: Limitations of the Transparency Ideal and Its Application to Algorithmic Accountability. New Media et Society, 20(3), 973989. doi: $10.1177 / 1461444816676645$

Bänziger, P. P., et Duttweiler, S. (2006). " Chère Marta, j'ai un problème : " la mise en mots du malaise sexuel dans le courrier du cœur. Revue des Sciences Sociales, 36, 108-115. 
Dix petits hacks Tinder : Les algorithmes au service d'une économie spéculative des rencontres amoureuses et sexuelles

Bardini, T., et Proulx, S. (2002). La culture du hack en ligne, une rupture avec les normes de la modernité. Les cahiers du numérique, 3(2), 35-54.

Béjin, A., et Pollak, M. (1977). La rationalisation de la sexualité. Cahiers internationaux de sociologie, 105-125.

Bergström, M. (2019). Les nouvelles lois de l'amour. Sexualité, couple et rencontres au temps du numérique. Paris: La Découverte.

Bowker, G. C. (2005). Memory Practices in the Sciences (Vol. 205). Cambridge: MIT Press.

boyd, D., et Crawford, K. (2012). Critical Questions for Big Data: Provocations for a Cultural, Technological, and Scholarly Phenomenon. Information, Communication et Society, 15(5), 662-679. doi: 10.1080/1369118X.2012.678878

Bucher, T. (2018). If... then: Algorithmic power and politics. Oxford: Oxford University Press.

Burrell, J. (2016). How the Machine 'Thinks': Understanding Opacity in Machine Learning Algorithms. Big Data et Society, 3(1), 1-12. doi: $10.1177 / 2053951715622512$

Cardon, D. (2003). Droit au plaisir et devoir d'orgasme dans l'émission de Menie Grégoire. Le Temps Des Médias, 1(1), 7794. doi: $10.3917 /$ tdm.001.0077

Crawford, M. (2004). Mars and Venus Collide: A Discursive Analysis of Marital Self-Help Psychology. Feminism and Psychology, 14(1), 63-79. doi: 10.1177/0959-353504040305

Cresswell, C. (2003). Mathematics and Sex. Crows Nest: Allen et Unwin.

Dourish, P. (2016). Algorithms and Their Others: Algorithmic Culture in Context. Big Data et Society, 3(2), 1-11. doi: $10.1177 / 2053951716665128$

Duguay, S. (2017). Dressing up Tinderella: Interrogating Authenticity Claims on the Mobile Dating App Tinder. Information, Communication et Society, 20(3), 351-367. doi: 10.1080/1369118X.2016.1168471 


\section{David Myles et Martin Blais}

Duguay, S., Burgess, J., et Light, B. (2017). Mobile Dating and Hookup App Culture. In Digital Media: Transformations in Human Communication. New York: Peter Lang.

Eubanks, V. (2018). Automating Inequality: How High-Tech Tools Profile, Police, and Punish the Poor. St. Martin's Press.

Foucault, M. (1975). Surveiller et punir. Paris: Gallimard.

Foucault, M. (1994). Dits et Écrits. 1954-1988, Paris: Gallimard.

Foucault, M. (2012). Du gouvernement des vivants: cours au Collège de France: 1979-1980. Paris: Gallimard.

Fry, H. (2015). The Mathematics of Love: Patterns, Proofs, and the Search for the Ultimate Equation. New York: Simon and Schuster.

Giddens, A. (1992). The transformation of intimacy: Sexuality, love and eroticism in modern societies. Cambridge: Polity Press.

Gillespie, T. (2014). The Relevance of Algorithms. In Gillespie, T., Boczkowski, P.J. et K.A. Foot (Éds), Media Technologies: Essays on Communication, Materiality, and Society (p. 167-194), Cambridge: MIT Press.

Gillespie, T. (2016). Algorithm. In Peters, B. (Éd.) Digital Keywords: A Vocabulary of Information Society and Culture (p. 18-30), Princeton: Princeton University Press.

Graham, L. J. (2011). The Product of Text and 'Other' Statements: Discourse Analysis and the Critical Use of Foucault. Educational Philosophy and Theory, 43(6), 663-674. doi: 10.1111/j.14695812.2010.00698.x

Gray, J. (1992). Men are from Mars. Women Are from Venus, New York: Harper Collins.

Haag, P. S. (1992). In Search of "the Real Thing": Ideologies of Love, Modern Romance and Women's Sexual Subjectivity in the US, 1920-40. Journal of the History of Sexuality, 2(4), 547-577.

Illouz, E. (2012). Pourquoi l'amour fait mal. L'expérience amoureuse dans la modernité. Paris : Seuil. 
Dix petits hacks Tinder : Les algorithmes au service d'une économie spéculative des rencontres amoureuses et sexuelles

Kitchin, R. (2017). Thinking Critically About and Researching Algorithms. Information, Communication et Society, 20(1), 14-29. doi: 10.1080/1369118X.2016.1154087

Krüger, S., et Spilde, A.C. (2019). Judging Books by Their CoversTinder Interface, Usage and Sociocultural Implications. Information, Communication et Society, 1-16. doi: 10.1080/1369118X.2019.1572771

Luhmann, N. (1986). Love as Passion: The Codification of Intimacy. Cambridge: Harvard University Press.

Massanari, A. (2017). \#Gamergate and The Fappening: How Reddit's Algorithm, Governance, and Culture Support Toxic Technocultures. New Media et Society, 19(3), 329-346. doi: 10.1177/1461444815608807

McGoey, L. (2012). Strategic Unknowns: Towards a Sociology of Ignorance. Economy and Society, 41(1), 1-16. doi: 10.1080/03085147.2011.637330

Ménard, M., Mondoux, A., Ouellet, M., et Bonenfant, M. (2016). Big data, gouvernementalité et industrialisation des médiations symboliques et politico-institutionnelles. Interfaces numériques, 5(2), 317-340.

Myles, D. (2020). Les rencontres amoureuses et sexuelles au temps des algorithmes: Une analyse comparative de Grindr et Tinder. In Piazzesi, C., Blais, M., Lavigne, J. \& C. Lavoie Mongrain (dir.). Intimités et sexualités contemporaines : Les transformations des pratiques et des représentations (p. 73-90), Montréal: Presses de l'Université de Montréal.

Neyland, D., et Möllers, N. (2017). Algorithmic IF... THEN. Rules and the Conditions and Consequences of Power. Information, Communication et Society, 20(1), 45-62. doi: 10.1080/1369118X.2016.1156141

Noble, S. U. (2018). Algorithms of Oppression: How Search Engines Reinforce Racism. Nyew York: NYU Press.

Pasquale, F. (2015). The Black Box Society. Harvard University Press. 


\section{David Myles et Martin Blais}

Rouvroy, A. (2014). Des données sans personne: le fétichisme de la donnée à caractère personnel à l'épreuve de l'idéologie des Big Data. Contribution en marge de l'Étude annuelle du Conseil d'État. Le numérique et les droits et libertés fondamentaux.

Rouvroy, A., et Berns, T. (2013). Gouvernementalité algorithmique et perspectives d'émancipation. Réseaux, 177(1), 163-196. doi: 10.3917/res.177.0163

van Dijck, J. (2014). Datafication, Dataism and Dataveillance: Big Data Between Scientific Paradigm and Ideology. Surveillance et Society, 12(2), 197-208. doi: 10.24908/ss.v12i2.4776

Velkova, J., et Kaun, A. (2019). Algorithmic Resistance: Media Practices and the Politics of Repair. Information, Communication et Society, 1-18. doi: 10.1080/1369118X.2019.1657162 